\title{
Directional Cuing of Target Choice in Human Smooth Pursuit Eye Movements
}

\author{
Siobhan Garbutt ${ }^{1,2}$ and Stephen G. Lisberger ${ }^{1,2,3}$ \\ ${ }^{1}$ Department of Physiology, ${ }^{2}$ Keck Center for Integrative Neuroscience, and ${ }^{3}$ Howard Hughes Medical Institute, University of California, San Francisco, San \\ Francisco, California 94143
}

\begin{abstract}
Perceptual attention and target choice for movement have many features in common. In particular, both generally are based on selection of a particular location in space. To ask whether motor control, like attention, also can exhibit target choice based on nonspatial features of the stimulus, we assessed the initiation of smooth pursuit eye movements when two targets move in different directions after human subjects have been cued which direction or color to track. The direction cue consisted of a patch of dots undergoing either $0 \%$ coherent motion or $50 \%$ coherent motion in the direction of motion of one of the subsequent targets. After a delay, the fixation spot was extinguished and two spots moved across the same small region of the visual field, one in the cued direction ("target") and one in an orthogonal direction ("distracter"). After the $0 \%$ coherent cue, pursuit was approximately the vector average of responses to the two motions presented singly. After the $50 \%$ coherent cue, the initial pursuit response was biased strongly toward the target that moved in the cued direction. The impact of the cued direction persisted over delays of up to $1000 \mathrm{~ms}$. Other cues about the direction of upcoming target motion biased the response similarly. Cues about target color also biased pursuit in the direction of motion of the cued target but were considerably less effective than cues indicating the direction of target motion. We conclude that target choice for movement, like perceptual attention, can be based on the features of the chosen target and not only its location in space.
\end{abstract}

Key words: motor planning; delay period; oculomotor; vector averaging; attention; motion processing

\section{Introduction}

Perceptual attention is based heavily on the spatial location of the attended target but also can be guided by cues about specific features of the target. Do these features of perceptual attention also apply to target choice for motor control? In smooth pursuit eye movements, for example, we already know that spatial cues dominate target choice. If a monkey makes a saccade to one of two identical moving targets, then postsaccadic eye velocity is controlled almost entirely by the motion of the target at the endpoint of the saccade (Gardner and Lisberger, 2001). Furthermore, direct tests of the spatial control of target choice indicate that it can be formalized as a spatial aperture that moves through visual space with the eye, perhaps preceding saccades slightly (Schoppik and Lisberger, 2006). In contrast, if monkeys or humans are presented with two identical targets that move in different directions without any cues about which target to track, then the initial presaccadic response approximates a vector average of the response evoked by each target alone (Lisberger and Ferrera, 1997; Ferrera, 2000; Sperling and Gegenfurtner, 2006).

Two kinds of previous approaches have set the stage for the

Received May 19, 2006; revised 0ct. 20, 2006; accepted 0ct. 23, 2006.

This work was supported by the Howard Hughes Medical Institute and National Institutes of Health Grant NS34835. We thankScott Ruffner for computer programming and Ken McGary for electronics. We are grateful to our three dedicated subjects.

Correspondence should be addressed to Siobhan Garbutt, University of California, San Francisco, Box 0444, 513 Parnassus Avenue, Room HSE-802A, San Francisco, CA 94143-0444. E-mail: sgarbutt@phy.ucsf.edu. DOI:10.1523/JNEUROSCI.4071-06.2006

Copyright $\odot 2006$ Society for Neuroscience $\quad$ 0270-6474/06/2612479-08\$15.00/0 project described here. First, studies of target choice for pursuit eye movements in humans have found that eye movement latencies are reduced more effectively by cues about the future location of the target in space than by cues about the direction of impending target motion (Adler et al., 2002). Monkeys can choose targets based on previous cues about the color of the rewarded target, but there is a substantial cost of increased movement latency (Ferrera and Lisberger, 1995). Second, studies of perceptual attention have shown that allocation of attention to a particular spatial location allows subjects to focus a disproportionate part of their neural resources on signals that arise from the cued part of space. As a result, thresholds are lower and processing is more acute for stimuli that fall in the attended locations (Posner, 1980). There are also examples of "feature-based" attention, in which subjects can direct the neural resources to objects of specific colors or shapes (Corbetta et al., 1990; Sàenz et al., 2003), as well as some evidence for "directional" attention, in which precuing about the relevant direction can enhance both perceptual reports about motion in that direction (Tsujimoto and Tayama, 2004) and the response gains of neurons in cortical motion middle temporal area MT (Maunsell and Treue, 2006). We now ask whether target choice for pursuit also can be based on nonspatial features of a moving target and, if so, for what class of features.

Our experiments precued human subjects about the direction of motion or the color of a target they were to track in the face of a distracter moving in a different direction. We studied only the "open-loop" initiation of pursuit that occurs before saccades, so that we could base our conclusions on a visually driven compo- 
nent of the movement yet exclude the powerful spatially based target choice that is expressed immediately after a saccade. We show that previous knowledge about the direction of the impending target motion creates a strong bias in presaccadic pursuit, whereas previous knowledge about the color of the target is less effective. Our results provide evidence that knowledge of the direction of impending target motion is a powerful cue for a form of target choice that can operate very early in a motor response, suggesting a motor analogy to feature-based perceptual attention.

\section{Materials and Methods}

The subjects were researchers from the Keck Center for Integrative Neuroscience at the University of California, San Francisco (UCSF): one female and two males, aged 29, 27, and 26 years. Subjects 1 and 3 had some previous experience as participants in eye movement experiments but were naive to the specific hypothesis being tested. Subject 2 was completely naive. All subjects were healthy and had normal vision. The Committee on Human Research, which is the Institutional Review Board for UCSF and its affiliates, approved all experimental procedures in advance, and the subjects gave their informed consent.

Subjects were seated on a cushioned chair with their heads stabilized using padded chin and forehead rests and a head strap. Stimuli were viewed binocularly, and the two-dimensional movements of the right eye were measured using the Fourward Technologies (Buena Vista, VA) Generation 6.1 Dual Purkinje Image Eye Tracker. Its specifications indicated that the spatial resolution of the eye tracker was $1 \mathrm{~min}$ of arc with a temporal resolution of $1 \mathrm{~ms}$, although the spatial resolution in practice seems much better than advertised. The automatic optical staging (auto stage) and focus servo of the tracker were disabled to avoid introducing head position artifacts into the eye position data. The eye position signal was low-pass filtered with a cutoff at $330 \mathrm{~Hz}$, and voltages proportional to eye velocity were obtained by differentiating the eye position signals with an analog circuit. The circuit differentiated signal content up to $25 \mathrm{~Hz}$ and rejected signals of higher frequencies.

\section{Pursuit stimuli}

In most experiments, visual stimuli were presented on a large analog oscilloscope (model A12-63; Xytron, Sylmar, CA) that was driven by the digital-to-analog outputs from a digital signal processing board in a Pentium computer. Temporal resolution of the display system was $4 \mathrm{~ms}$, and nominal spatial resolution was $2^{16}$ pixels. The monitor was $40 \mathrm{~cm}$ from the subject and subtended a visual angle of $\sim 47^{\circ}$ horizontally by $40^{\circ}$ vertically. Pursuit targets were small white spots $\left(0.3^{\circ}, \sim 20 \mathrm{~cd} / \mathrm{m}^{2}\right)$ presented on a dark background $\left(<0.001 \mathrm{~cd} / \mathrm{m}^{2}\right)$. In the experiments that required color, the visual stimuli were generated by a visual stimulus generator frame buffer (Cambridge Research Systems, Kent, UK) and displayed on a Barco (Kortrijk, Belgium) color monitor that subtended $46^{\circ}$ horizontally and $31^{\circ}$ vertically at $40 \mathrm{~cm}$ from the subject. Pursuit targets were $0.3^{\circ}$ red $\left(\sim 12 \mathrm{~cd} / \mathrm{m}^{2}\right)$, green $\left(\sim 35 \mathrm{~cd} / \mathrm{m}^{2}\right)$, or white $(\sim 48$ $\left.\mathrm{cd} / \mathrm{m}^{2}\right)$ spots presented on a dark background $\left(<0.001 \mathrm{~cd} / \mathrm{m}^{2}\right)$. Temporal resolution of the display system was $60 \mathrm{~Hz}$ and spatial resolution was $1280 \times 1024$ pixels. Experiments were conducted in a dimly lit room. Data were collected in individual sessions that lasted $45 \mathrm{~min}$ to $1 \mathrm{~h}$ and included some breaks between blocks. The eye position signals were calibrated at the beginning of each recording session by having the sub- ject fixate stationary targets at known horizontal and vertical eccentricities.

Trials were variations on a basic task in which the subject selected one of two moving targets as a pursuit target based on a previous cue. The temporal sequence of a single trial is illustrated in Figure $1 \mathrm{~A}$. Trials began with an initial fixation period of $600-1000 \mathrm{~ms}$, followed by a cue that was present for 600-1200 ms. After the cue disappeared, there was a "delay" period of $0-1000 \mathrm{~ms}$. The fixation target remained on throughout the cue and delay periods, and the subject was required to fixate within a $2^{\circ}$ window around the target for the entire time it was illuminated. If fixation was broken before the end of the delay period, then the trial was aborted. If fixation was maintained, then the fixation target was extinguished and replaced immediately with one or two moving spots. When the pursuit stimulus comprised two moving spots, one was a target that would move for 700-900 ms, and one was a distracter that disappeared after moving for $300 \mathrm{~ms}$. On randomly interleaved trials, a single target appeared and moved for 700-900 ms without a distracter. We created step ramp motion (Rashbass, 1961) to minimize the occurrence of saccades during the initiation of pursuit by having both targets appear $2^{\circ}$ eccentric and undergo ramp motion that took them toward the fixation point at $10 \%$ s.

\section{Experimental design}

Experiment 1. The cue was a patch of dynamic random dots that measured $10 \times 10^{\circ}$ and was centered on the fixation target, which remained on during the presentation of the cue. We used cues in which $50 \%$ of the dots moved coherently and 50\% moved randomly because it was easy for subjects to perceive the direction of motion within the patch, but they did not track the motion (as they did for 100\% correlated dots). The starting locations of the dots within the patch were assigned randomly, and 60 dots were visible at any one time. Each dot moved at a speed of $10 \%$. The frame period during the presentation of the cue was $16 \mathrm{~ms}$; every $16 \mathrm{~ms}$, each dot had a $50 \%$ chance of continuing on its path and a $50 \%$ chance of 
being randomly repositioned. The subjects were instructed to follow the target that moved in the same direction as the majority of dots in the cue. In experiment 1 , the identical-looking target and distracter moved orthogonally; when the target moved horizontally, the distracter moved vertically and vice versa (Fig. $1 A$ ). In any given trial, the cue was randomly chosen to comprise horizontal or vertical motion. On $70 \%$ of trials, the direction of motion in the cue was "valid" and corresponded to the direction of motion of the spot that would ultimately become the "target" and remain visible through 700-900 ms of motion. On the other $30 \%$ of trials, the cue was intentionally "invalid" and corresponded to the direction of motion of the spot that ultimately would become the "distracter" and remain visible for only $300 \mathrm{~ms}$ of motion. Comparison of the responses for a given cue when it was valid versus invalid revealed identical eye velocities until at least $100 \mathrm{~ms}$ after the distracter disappeared, which corresponded to $400 \mathrm{~ms}$ after the onset of target motion. This validates our choice (see below) of eye velocity up to $300 \mathrm{~ms}$ after the onset of target motion as an analysis interval in which eye velocity was driven by the visual stimulus and modulated according to the direction indicated by the cue. Because subject 1 had marginally better rightward pursuit than leftward and downward pursuit than upward, the orthogonal directions chosen for subject 1 were right and down. The orthogonal directions presented to subject 2 were left and up. Those for subject 3 were right and up. All data have been rotated so that the directions can be presented as though they had been rightward and upward.

As a control, in a separate experiment, we ran trials with a cue that consisted of $0 \%$ coherent motion, so that the patch contained no net motion in any direction. In experiments using the $0 \%$ coherent cue, one or two targets were present in 70 or $30 \%$ of the trials, respectively. The subjects were instructed that they were free to select either of the stimuli to follow in two-target trials.

Experiment 2. In separate experimental sessions, subjects were presented with one of the following different cues (Fig. 1 B): a $50 \%$ coherent "direction" cue as in experiment 1, a "color-target" cue indicating the color of the intended target, a "color-direction" cue indicating the direction of the intended target, or a "destination" cue. The destination cue consisted of a $5 \times 5^{\circ}, 0 \%$ coherent patch of dots presented so that the center of the patch was $5^{\circ}$ to the left or right of the vertical meridian. The direction of the offset (left or right) cued the subject about the side of the visual field where the upcoming target stimulus would stop moving. Because stimuli started on one side of the visual field and moved across the original fixation point to the other side, this provided an unambiguous cue about the direction (and initial location) of the upcoming target motion. The color-target cue was created by changing the color of the fixation target to red or green for 600-1200 ms during the fixation period (after Ferrera and Lisberger, 1995). This cue indicated the color of the target we wanted the subject to pursue but gave no information about the direction of the impending motion of the target stimulus. For each trial, the color of the target and its direction were selected at random. For the color-direction cue, subjects were instructed to follow the spot ramping right if the cue was red and the spot ramping left if the cue was green; the target and distracter were white. Finally, in one experiment done on a single subject, we used a tone as a cue; the subject was instructed to track the rightward target motion if the tone was presented during the cue interval and the leftward target motion if the tone was not presented.

In experiment 2, the target and distracter moved horizontally; when the target moved rightward, the distracter moved leftward and vice versa. We chose to use opposite directed target and distracters along the horizontal meridian for three reasons: (1) horizontal pursuit was better than vertical pursuit in two of our three subjects, and we wanted to work with balanced responses to the two stimuli; (2) the display screen was wider than it was high, allowing greater scope for stimulus presentation; and (3) for the color-direction cue, it would be easier for the subjects to remember that the two colors corresponded to opposite versus orthogonal directions of motion. The presence of minor left-right asymmetries in our subjects did not create any noteworthy effects on their responses in experiment 2 .

\section{Data analysis}

We selected the eye movements in the first $300 \mathrm{~ms}$ after the onset of target motion for analysis, because this was the interval when both the target and distracter were present. Approximately $17 \%$ of trials included a saccade and/or eye blink in this interval and were excluded from analysis. The responses to identical stimuli then were aligned on the onset of target motion, and the mean and SD of horizontal and vertical eye velocity were calculated at each sample point. All data points are based on averages of 10-40 repetitions of each trial type.

We used the time averages of eye velocity to calculate the latency, initial eye acceleration, and eye velocity at different times after target motion onset. We defined the time of the onset of pursuit by the intersection of regressions along the baseline eye velocity and the initial part of the pursuit response (after Carl and Gellman, 1987). The baseline was defined as the period beginning $100 \mathrm{~ms}$ after the fixation target was presented and ending when it was extinguished. The initial part of the response was defined as the $50 \mathrm{~ms}$ after the time when the amplitude of eye velocity exceeded 3 SDs from baseline. Latency was computed as the time interval from the onset of target motion to the intersection of these two regression lines. We then computed the initial eye acceleration as the change in eye velocity during the first $100 \mathrm{~ms}$ after the eye velocity trace exceeded three times the SD during the baseline interval, divided by 100 $\mathrm{ms}$. Finally, we computed the velocity of pursuit 150, 200, 250, and 300 $\mathrm{ms}$ after the onset of target motion as the average within a $20 \mathrm{~ms}$ interval that bracketed each time point.

\section{Results}

Effect of a directional motion cue on the initiation of pursuit

We assessed the effect of the cue by presenting stimuli that comprised a target and distracter moving in orthogonal directions and comparing the eye velocity responses when the direction of the target was cued versus those when a cue was present but did not contain any coherent motion. In Figure $2 A$, for example, the cued trials indicated that the subject should attempt to track the horizontal target motion even when the distracter moved vertically. Averaged traces of eye velocity as a function of time for subject 2 (Fig. $2 A$ ) show that horizontal eye velocity was largest when the pursuit stimulus was a single horizontal target motion, intermediate when the cue was given, and smallest when a cue was present but did not contain any coherent motion. Vertical eye velocity showed the opposite effects. It was largest when the cue was present but did not contain any coherent motion, intermediate when the cue indicated that the subject should track the horizontal target motion, and smallest for the horizontal motion of a single spot.

Figure 2, $A$ and $B$, shows the eye velocity for $300 \mathrm{~ms}$ after the onset of target motion. These represent saccade-free pursuit because trials were included in the average only if they were not contaminated by saccades within this $300 \mathrm{~ms}$ interval. Comparison of the differently colored traces used to show data from different conditions reveals that the effects of the cue appeared quite soon after the initiation of pursuit, in some cases immediately. In Figure 2, the responses to single targets were obtained from trials in which the target motion was preceded by a valid cue of $50 \%$ coherent motion. These responses were not significantly different from those to single targets after a cue that did not contain coherent motion or from those to single targets presented without a cue. However, invalid cues $(50 \%$ coherent motion in the direction orthogonal to target motion) did have a small effect on the responses to single targets; across subjects and directions of target motion, latencies to the initiation of pursuit were 15-23 ms longer, initial eye acceleration was reduced by $7-24 \%$, and eye velocity at $300 \mathrm{~ms}$ was reduced by $20-26 \%$.

The same data are plotted in Figure $2 C$ in a way that compares the trajectory of eye motion for the different target and cuing 
A Horizontal cue

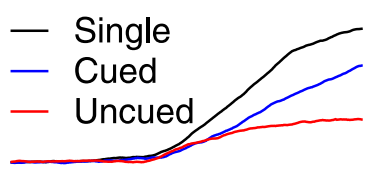

Horizontal eye velocity
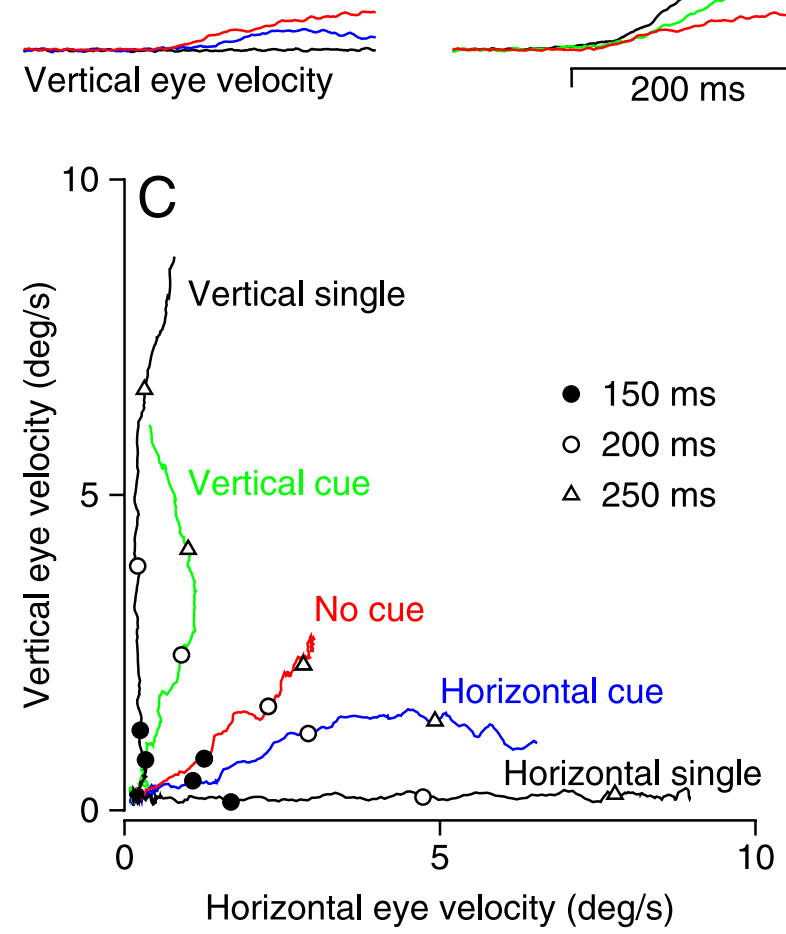

Figure 2. Effect of a directional cue on the responses of a representative subject to the orthogonal motion of a target and distracter. $\boldsymbol{A}, \boldsymbol{B}$, Averages of horizontal (top traces) and vertical (bottom traces) eye velocity when the cue provided horizontal $(\boldsymbol{A})$ or vertical $(\boldsymbol{B})$ motion. $\boldsymbol{C}$, Data from $\boldsymbol{A}$ and $\boldsymbol{B}$ are replotted, now showing the trajectory of eye velocity in two dimensions. Time is represented by the symbols on the traces: filled circles, open circles, and open triangles show moments 150,200, and $250 \mathrm{~ms}$ after the onset of target motion. Traces are as follows: black, responses to single targets; red, response when the cue contained no overall motion; blue and green, cue indicated that impending target motion would be horizontal or vertical, respectively. Data are from subject 2.

conditions. Here, vertical eye velocity is plotted versus horizontal eye velocity for each of the conditions used in Figure 2, $A$ and $B$. In each trial, average eye velocity started at the origin of the graph $(0,0)$ and, as the response evolved, moved in a consistent trajectory rightward and/or upward. The responses at different times can be compared across conditions by looking at the symbols on the traces, which indicate eye velocity 150,200, and $250 \mathrm{~ms}$ after the onset of target motion. The trajectory depended strongly on the stimulus conditions. The horizontal or vertical motion of single targets evoked trajectories of eye motion that were almost perfectly horizontal or vertical (black traces). When the cue did not contain coherent motion ("no cue", red trace), the trajectory of eye velocity was in a direction that was close to $45^{\circ}$, as expected if the subjects were pursuing the vector average of the responses to the target and distracter singly (Lisberger and Ferrera, 1997). When the cue contained horizontal or vertical motion (blue and green traces), the trajectory of the responses deviated away from the prediction of vector averaging toward the cued direction.
These effects were visible even $150 \mathrm{~ms}$ after the onset of target motion (filled circles) and were large and consistent both 200 and $250 \mathrm{~ms}$ (open circles and open triangles) after the onset of target motion.

Figure 3 plots the responses to the orthogonal motion of two spots, in which each individual symbol summarizes data for one delay period in one experiment. The top graphs (Fig. $3 A-C$ ) plot vertical eye velocity as a function of horizontal eye velocity, both measured $300 \mathrm{~ms}$ after the onset of target motion. The bottom graphs (Fig. 3D-F) plot initial vertical eye acceleration as a function of initial horizontal eye acceleration. Both measures represent presaccadic pursuit. In each graph, the data for the $0 \%$ coherent cue (open circles) plot in an intermediate location, indicating that the response comprised both horizontal and vertical components. For subject 1 , the response to two targets after the $0 \%$ coherence cue was biased toward horizontal eye motion (average direction $21^{\circ}$ above horizontal); for subject 2, the response after the $0 \%$ coherence cue was more equally horizontal and vertical; and for subject 3 , it was biased toward vertical $\left(62^{\circ}\right.$ above horizontal). These biases may reflect individual differences in responses in the two-target task, but the important point is that directional cues biased the response of each subject toward the direction of motion indicated by previous cues. For all subjects, and both measures of pursuit, the cue overcame or enhanced the baseline bias so that the response was dominated by horizontal or vertical eye motion according to whether the $50 \%$ coherence cue comprised horizontal or vertical target motion (filled triangles vs filled circles).

\section{Effect of spatial uncertainty on directional cuing}

In the experiments summarized by Figures 2 and 3, the two targets started at spatial locations that differed by $2.8^{\circ}$ : one started $2^{\circ}$ to the left or right of fixation and one started $2^{\circ}$ above or below fixation. This was necessary because the targets had to move in different directions but directly toward the position of fixation to avoid early saccades and provide a long-enough interval of presaccadic pursuit for analysis. To determine whether the small difference in spatial location was being used to choose the relevant target and give the appearance of directional cuing, experiments in subject 3 first were performed using targets that started randomly at two different pairs of locations within the $2 \times 2^{\circ}$ square below and to the left of fixation. The spatial uncertainty prevented the subject from using spatial location to choose the target moving in the correct direction. Still, we were able to analyze only the data when the rightward and upward moving targets started $2^{\circ}$ to the left and below fixation because of the prevalence of early saccades with other starting locations. Figure 4 uses the same format as Figure $2 \mathrm{C}$ to show that the effect of the directional cue was just as strong when the spatial location of the moving targets was uncertain (blue traces) as it was in later experiments when the spatial location was certain (black traces). However, comparison of the eye velocities at the times indicated by the symbols on the traces indicates that a given eye velocity was achieved somewhat later on the day of the first experiment, with spatial uncertainty, than on the day of the second experiment, without spatial uncertainty. We have no way to return this subject to the fully naive state of day 1 to address the cause of this difference in the gain of the responses.

\section{Effects of the duration of the delay period}

In some of the experiments, we used eight delay durations between 0 and $1000 \mathrm{~ms}$ so that we could assess any effects of the duration of the delay period on the latency or magnitude of pur- 

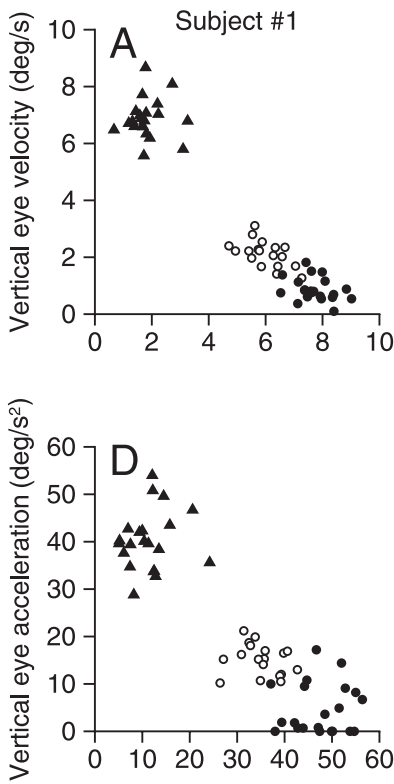
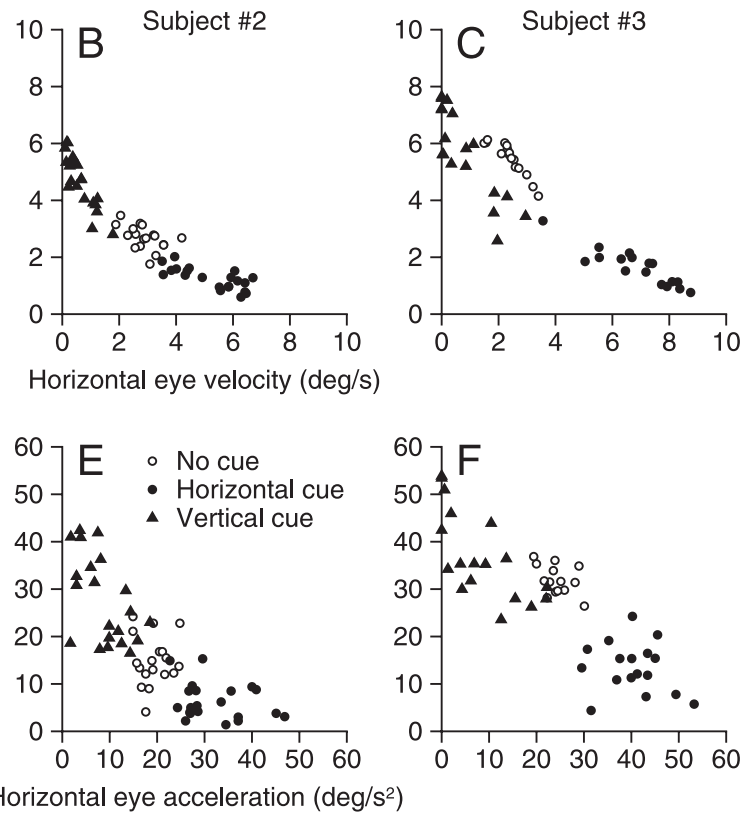

Figure 3. Quantitative analysis of the effect of directional cues on presaccadic target choice. Each column shows a pair of graphs for one of our subjects. Each data point represents the horizontal and vertical eye velocity $300 \mathrm{~ms}$ after target onset $(\boldsymbol{A}-\boldsymbol{C})$ or the initial eye acceleration $(\boldsymbol{D}-\boldsymbol{F})$ for a different experiment and delay periods. Open circles, filled triangles, and filled circles summarize the pursuit after a $0 \%$ coherent cue (no-cue), a vertical cue, and a horizontal cue. Data were obtained from three experiments and delay durations ranging from 0 to $1000 \mathrm{~ms}$ in each subject.

suit responses. To assess the effect on the relative weighting of the cued target versus the distracter, we solved the following two equations in two unknowns:

$$
\begin{aligned}
& \dot{E}_{\mathrm{H}}^{\mathrm{TD}}=w_{\mathrm{T}} \dot{E}_{\mathrm{H}}^{\mathrm{T}}+w_{\mathrm{D}} \dot{E}_{\mathrm{H}}^{\mathrm{D}} \\
& \dot{E}_{\mathrm{V}}^{\mathrm{TD}}=w_{\mathrm{T}} \dot{E}_{\mathrm{V}}^{\mathrm{T}}+w_{\mathrm{D}} \dot{E}_{\mathrm{V}}^{\mathrm{D}},
\end{aligned}
$$

where $w_{T}$ and $w_{D}$ are the weights afforded the motion of the target and distracter to account for the recorded eye movements, and $\dot{E}_{Y}^{X}$ represents the eye velocity recorded for the motion of targets $x$ along axis $y$; T, D, H, and $\mathrm{V}$ refer to target, distracter, horizontal, and vertical, respectively. The weights consistently favored the target when a valid cue was provided (Fig. $5 A$, filled symbols) and afforded relatively low weight to the distracter (open symbols). Importantly, the total of the two weights (dashed curve) was consistently less than one by $\sim 25 \%$, indicating that the overall pursuit response was weaker when two targets were present than for a single target, despite the presence of valid cues. A similar reduction in the overall pursuit response appeared for two-target stimuli with the $0 \%$ coherent "cue": for our three subjects, the sum of the weights afforded the two targets was $0.72,0.52$, and 0.71 . Thus, the reduction in the strength of the initiation of pursuit was related to the presence of two targets and not to the use of a cue.

The latency of the initiation of pursuit depended strongly on the duration of the delay period for both single and two-spot stimuli (Fig. 5B). Averaged across subjects, latency was nearly 200 ms when the duration of the delay period was 0 , decreased to almost $100 \mathrm{~ms}$ when the duration of the delay was $200 \mathrm{~ms}$, and then reached an asymptote at an intermediate value of $\sim 140 \mathrm{~ms}$ for the longest delays. Because of the longer latency to initiate pursuit, eye velocity measured $300 \mathrm{~ms}$ after the onset of target motion increased as delay increased from 0 to $200 \mathrm{~ms}$, and then was steady for delay periods up to $1000 \mathrm{~ms}$ (Fig. 5C). Initial eye acceleration did not depend on the duration of the delay period
(Fig. 5D). The differential effects on eye velocity $300 \mathrm{~ms}$ after the onset of target motion and initial eye acceleration would be expected because the former is referenced to the onset of target motion, whereas the latter is referenced to the onset of eye motion, which itself varied consistently as a function of the duration of the delay period.

Comparison of efficacy of different cues The data presented so far indicate that a directional cue provided by a moving stimulus is able to bias the subsequent response of the pursuit system strongly in the direction of the cue. Next, we evaluated what features make a cue effective. Four different types of visual cues were used in different recording sessions on different days. Three cue types indicated intended target direction in different ways: (1) the direction cue was the same $50 \%$ coherent patch of moving dots used so far; (2) the destination cue was a $0 \%$ coherent patch of dots placed on the right or left of the fixation spot to cue the side to which the target would move (opposite the side where the target would appear); and (3) the colordirection cue was red when subjects were to track the spot that ramped to the right and green when the intended target ramped to the left. The fourth, the color-target cue, indicated the color of the target the subject was intended to track but did not indicate the direction of motion of the cued target.

Figure 6 shows averages of the horizontal eye velocity induced by each cue. In these experiments, the two spots moved to the left and right along the horizontal meridian. To present the results, we inverted all responses when the cued target moved to the left, so that traces would deflect upward when the subject tracked in the direction of the cued target. We then averaged responses together for leftward and rightward cues. The averages indicate that any cue of the impending direction of motion of the tracking target is equally effective at biasing human subjects to track in the cued direction. Thus, the eye velocity traces overlapped for the direction cue (green), the destination cue (cyan), and the colormotion cue (dark blue). In contrast, the cue that indicated the color of the target to track rather than its direction of motion (color cue, magenta traces) biased the initiation of pursuit only slightly more than the weak response found when the direction cue was a $0 \%$ coherent patch of dots presented at fixation (red traces). All traces in Figure 6 represent averages across delay periods and direction, and each was derived from 200 to 300 trials, except for the no-cue data, which were derived from $\sim 40$ trials.

To ask whether the effects of the visual cues used above reflected target choice based on the anticipated direction of the target rather than a visually based priming of the inputs for smooth pursuit, we conducted an additional experiment in which a tone was used as a cue. In this experiment, subject 2 was instructed to follow the target ramping to the right if a tone was presented during the cue period and to follow the target to the left if the tone was not presented. The effect of the tone cue was identical to those of the visual cues that indicated the impending direction of motion of the target the subject was intended to choose (Fig. 6B). 


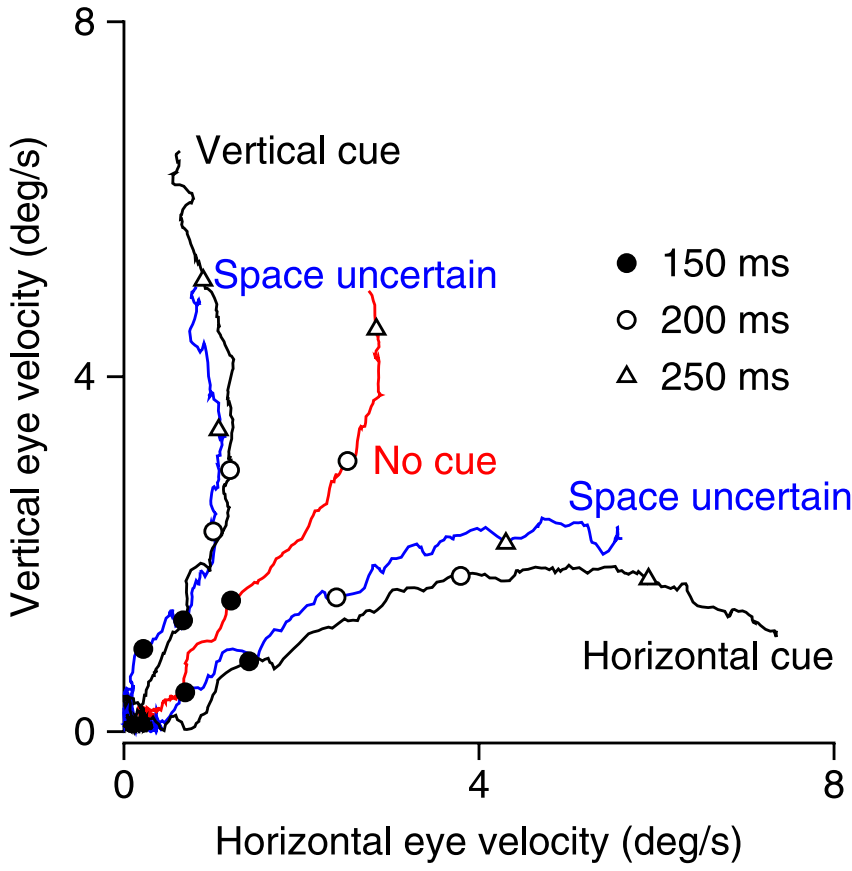

Figure 4. Test of whether directional cues were being used to choose pursuit targets based on their spatial location. Time vectors of horizontal and vertical eye velocity have been plotted in a way that shows the trajectory of eye velocity in two dimensions. Time is represented by the symbols on the traces: filled circles, open circles, and open triangles show moments 150, 200, and $250 \mathrm{~ms}$ after the onset of target motion. Traces are as follows: black, responses in experiments in which the spatial location of the target could be determined by the directional cue; red, responses when the cue contained no overall motion; blue, responses in experiments in which the spatial location of the target was uncertain and was not knowable from the directional cue. Data are from subject 3 . Experiments with uncertain target locations were run before those with certain target locations.

\section{Discussion}

In spatial vision, the brain is confronted with a huge expanse of visual field to monitor in the interest of recognizing and reacting to potentially subtle features of the scene at specific locations. An advantage is conferred to a specific location in space if the subject is precued to direct perceptual attention to that location (Posner, 1980). Spatial attention predominates in perceptual processing, although subjects can attain some advantage if cued to the features of the impending stimulus rather than to its likely spatial location (Corbetta et al., 1990; Sàenz et al., 2003). In the present paper, we analyze potential correlates to perceptual attention in the features of target choice for voluntary movement. Our data show that the features of a target rather than simply its spatial location can play an important role in target choice. When subjects were cued about the direction of an impending target motion, the direction of the initiation of smooth pursuit eye movements was biased strongly toward a target moving in the cued direction, despite the presence of an identical target moving in a different direction across the same restricted locus of the visual field. This effect is superimposed on a slight reduction in the strength of the initiation of pursuit that appears for all two-target stimuli, without regard for whether a cue is presented.

Our finding of strong target selection in the presaccadic initiation of pursuit suggests changes in the view of pursuit target choice given by our previous reports, which have emphasized differences in target choice between presaccadic and postsaccadic pursuit. When animals are not cued, presaccadic pursuit is approximately the vector average of the responses to the two targets presented singly (Lisberger and Ferrera, 1997), but postsaccadic
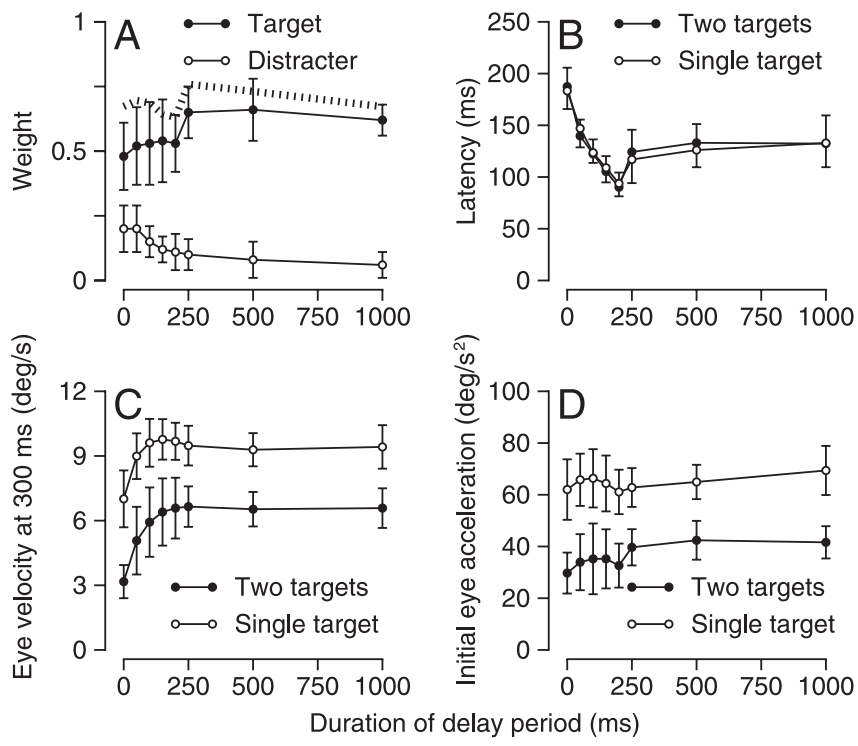

Figure 5. Effect of delay duration on the responses to one and two moving spots after $50 \%$ coherent cues. $\boldsymbol{A}$, Relative weighting of target and distracter when both are present. Filled and open symbols show the weighting afforded the direction of motion of the target and distracter, respectively. The dashed line represents the sum of the weightings for the target and the distracter. $\boldsymbol{B}$, Latency for the initiation of pursuit. $\boldsymbol{C}$, Magnitude of eye velocity $300 \mathrm{~ms}$ after the onset of target motion. $\boldsymbol{D}$, Eye acceleration in the initial $100 \mathrm{~ms}$ after the onset of pursuit. In $\boldsymbol{B}-\boldsymbol{D}$, filled and open symbols show the responses to target/distracter stimuli and single targets, respectively. Data were pooled across subjects and spot direction. Error bars show SDs of the mean across subjects.

pursuit strongly favors the target at the endpoint of the saccade (Lisberger, 1988; Gardner and Lisberger, 2001). We know that the postsaccadic effects are a consequence of target choice and not simply the predominance of foveal vision: the chosen target is tracked immediately after the saccade, at least $50 \mathrm{~ms}$ before there has been any chance for foveal visual inputs to influence pursuit; furthermore, the act of executing the saccade seems to be sufficient to generate target choice (Gardner and Lisberger, 2002). The data in the present report emphasize that presaccadic target choice also can be strong if the behavioral task is designed to bring out this feature of pursuit.

Was presaccadic target choice in our subjects controlled by presetting the pursuit system to respond more favorably in a given direction? Or, alternatively, was the direction cue given to our subjects used to infer the likely spatial location of the target moving in the cued direction and then used to co-opt a spatial mechanism of target choice? We believe that the directional cue was used to cue direction rather than space because, in the experiments that used orthogonal target motions, the target and distracter moved across the same $2 \times 2^{\circ}$ patch of visual field. Although the neural effects of perceptual attention can resolve different targets within such a small spatial range (Treue and Maunsell, 1999), target choice for pursuit seems to be based on a spatial filter that is much broader, covering in excess of $10^{\circ}$ of visual space (Schoppik and Lisberger, 2006). Furthermore, in the one subject we tested, uncertainty about the spatial location of the two targets did not reduce the effect of the cue on the direction of pursuit. Because this subject was naive about the experiment when tested with uncertainty about the spatial location of the cued target, we conclude that he (and the other subjects) were biasing the pursuit system in favor of the cued direction of motion and not the expected spatial location of the target that would move in the cued direction. 

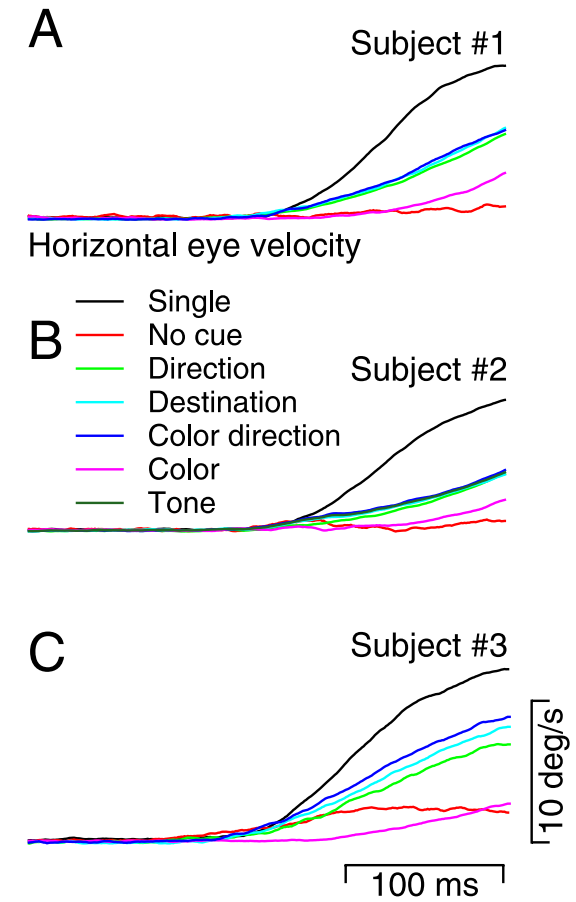

Figure 6. Effect of different types of cues on target choice in the initiation of pursuit. Each panel shows averages of horizontal eye velocity as a function of time, and the different panels show responses of our three subjects. Traces are averaged over delay periods and direction (right vs left). Colors indicate the following: black, single targets; red, $0 \%$ coherent cue; green, $50 \%$ coherent direction cue; cyan, stationary destination cue; dark blue, color cue indicating which direction to track; magenta, color cue indicating which color target to track; deep green, tone cue. Note that it is difficult to distinguish the green, cyan, blue, and deep green traces because they lie on top of each other.

We were motivated to look for an effect of cuing the direction of target motion to develop a paradigm that would allow investigation of the neural mechanisms of motor planning for pursuit. Previous studies have provided some evidence for motor planning but were either only partially successful or operated in regimens that seemed poorly adaptable to analysis of the neural correlates of the planning. For example, Ferrera and Lisberger (1995) used a color cue to induce monkeys to choose one of two targets before the first saccade but were successful only at the cost of at least $50 \mathrm{~ms}$ of added latency in the pursuit response. Analysis of pursuit latency has been more successful at a behavioral level but has not suggested strong neural recording experiments. Adler et al. (2002) used a spatial cue to shorten the latency for the target moving at the cued location but did not analyze the subsequent trajectory of eye velocity. Also, it is known that imposition of a temporal gap between the offset of the fixation point and the onset of an eccentric moving target reduces the latency for the initiation of pursuit (Krauzlis and Miles, 1996; Knox, 1998). Interestingly, a gap of 200-300 ms caused the largest decrease in latency (Krauzlis and Miles, 1996), in good agreement with the duration of the delay period that led to the greatest decrease in latency in our experiments.

Finally, effects similar to those reported here are present in the anticipatory pursuit that precedes target motion when human subjects are cued about the impending target motion. Jarrett and Barnes (2001) found that previous directional cues could be used to generate anticipatory pursuit responses. Anticipatory responses even can be scaled to the expected target velocity (Jarrett and Barnes, 2001; Poliakoff et al., 2005). Thus, previous reports also give evidence of the existence of a mechanism of target choice in pursuit. Our results go much further, however, by elucidating mechanisms of target choice in a time interval that is early enough to be before the first catch-up saccade but late enough so that smooth eye movement is driven by visual motion rather than by anticipation and expectation. The presence of a strong effect of cuing on the visually driven initiation of pursuit provides a basis for future analysis of the neural representations and mechanisms of target choices for motor control.

In most other motor systems, the use of delay tasks has revealed the accumulation of neural activity in the parietal and motor cortices during the interval between the onset of planning for the movement and its actual execution (Bruce and Goldberg, 1985; Kettner et al., 1996). Neural activity during delay periods has proven to have interesting features in conditions such as the countermanding task, in which saccades must be executed or cancelled based on cues delivered at different times after a saccade target appears (for review, see Schall et al., 2002) and in creating the neural basis for decisions (Shadlen and Newsome, 2001). Two features of our data imply that significant planning can occur in a delay period for smooth pursuit eye movements: pursuit latencies are shorter in the presence of a delay period, and target choice is enhanced somewhat with non-zero delay periods. We expect that the presence of delay activity will be a feature of the responses of neurons in the frontal pursuit area. Delay activity might also be diagnostic for neurons that are involved in pursuit in complex areas with many functions, such as the medial superior temporal area MST in the parietal cortex.

Our data highlight the emergence of increasingly strong parallels between perceptual attention and target choice for voluntary movements. Previous work has already shown that postsaccadic pursuit chooses targets according to their location in space (Schoppik and Lisberger, 2006), in agreement with the primacy of space for perceptual attention (Posner, 1980). Now, we demonstrated that cues about a feature of the visual stimulus, its impending direction of motion, have a strong influence on the initial pursuit response to motion that comprises targets moving in two different directions. Feature-based target choice seems similar to feature-based attention. For the processing of visual motion, extrastriate area MT is a major source of visual signals and drives both perceptual and pursuit behavior (Newsome et al., 1985; Newsome and Pare, 1988). The gains of response tuning functions of neurons in MT are subject to approximately equal modulation by either spatial or feature-based attention (Maunsell and Treue, 2006). If target choice for pursuit eye movements had access to the same mechanisms that allow perceptual attention to modulate the responses of MT neurons, then the representation of motion in MT would be biased toward the motion in the cued direction in the paradigm we developed. Thus, perceptual attention and target choice for voluntary movements may have parallels not only in the nature of the cues that modulate behavior but also in the neural circuits that are subject to modulation.

Finally, we think it is noteworthy that the most successful feature-based cues for target choice in pursuit have the modality (direction) that is most relevant to the movement itself. The function of pursuit is to rotate the eyes at the speed and direction of target motion, without great concern for any differences between eye and target position. Throughout the brain, signals that drive pursuit are coded in terms of the direction and speed of target or eye motion. Thus, it makes sense that presaccadic target choice for pursuit could be based strongly on directional target choice, more so than other movements that are more concerned with 
spatial location and might therefore be dominated by spatial mechanisms of movement choice.

\section{References}

Adler SA, Bala J, Krauzlis RJ (2002) Primacy of spatial information in guiding target selection for pursuit and saccades. J Vis 2:627-644.

Bruce CJ, Goldberg ME (1985) Primate frontal eye fields. I. Single neurons discharging before saccades. J Neurophysiol 53:603-635.

Carl JR, Gellman RS (1987) Human smooth pursuit; stimulus-dependent responses. J Neurophysiology 57:1446-1463.

Corbetta M, Miezin FM, Dobmeyer S, Shulman GL, Petersen SE (1990) Attentional modulation of neural processing of shape, color, and velocity in humans. Science 248:1556-1559.

Ferrera VP (2000) Task-dependent modulation of the sensorimotor transformation for smooth pursuit eye movements. J Neurophysiol 84:2725-2738.

Ferrera VP, Lisberger SG (1995) Attention and target selection for smooth pursuit eye movements. J Neurosci 15:7472-7484.

Gardner JL, Lisberger SG (2001) Linked target selection for saccadic and smooth pursuit eye movements. J Neurosci 21:2075-2084

Gardner JL, Lisberger SG (2002) Serial linkage of target selection for orienting and tracking eye movements. Nat Neurosci 5:892-899.

Jarrett CB, Barnes G (2001) Volitional selection of direction in the generation of anticipatory ocular smooth pursuit in humans. Neurosci Lett 312:25-28.

Kettner RE, Marcario JK, Port NL (1996) Control of remembered reaching sequences in monkey. II. Storage and preparation before movement in motor and premotor cortex. Exp Brain Res 112:347-358.

Knox PC (1998) Stimulus predictability and the gap effect on pre-saccadic smooth pursuit. NeuroReport 9:809-812.

Krauzlis RJ, Miles FA (1996) Decreases in the latency of smooth pursuit and saccadic eye movements produced by the "gap paradigm" in the monkey. Vision Res 36:1973-1985.

Lisberger SG (1998) Postsaccadic enhancement of initiation of smooth pursuit eye movements in monkeys. J Neurophysiol 79:1918-1930.
Lisberger SG, Ferrera VP (1997) Vector averaging for smooth pursuit eye movements initiated by two moving targets in monkeys. J Neurosci 17:7490-7502.

Maunsell JH, Treue S (2006) Feature-based attention in visual cortex Trends Neurosci 29:317-322.

Newsome WT, Pare EB (1988) A selective impairment of motion perception following lesions of the middle temporal visual area (MT). J Neurosci 8:2201-2211.

Newsome WT, Wurtz RH, Dursteler MR, Mikami A (1985) Deficits in visual motion processing following ibotenic acid lesions of the middle temporal visual area of the macaque monkey. J Neurosci 5:825-840.

Poliakoff E, Collins CG, Barnes GR (2005) Attention and selection for predictive smooth pursuit eye movements. Brain Res Cogn Brain Res 25:688-700.

Posner MI (1980) Orienting of attention. Q J Exp Psychol 32:3-25.

Rashbass C (1961) The relationship between saccadic and smooth tracking eye movements. J Physiol (Lond) 159:326-338.

Sàenz M, Buracas GT, Boyton GM (2003) Global feature-based attention for motion and color. Vision Res 43:629-637.

Schall JD, Stuphorn V, Brown JW (2002) Monitoring and control of action by the frontal lobes. Neuron 36:309-322.

Schoppik DS, Lisberger SG (2006) Saccades exert spatial control of motion processing for smooth pursuit eye movements. J Neurosci 26:7607-7618.

Shadlen MN, Newsome WT (2001) Neural basis of a perceptual decision in the parietal cortex (area LIP) of the rhesus monkey. J Neurophysiol 86:1916-1936

Spering M, Gegenfurtner KR, Kerzel D (2006) Distractor interference during smooth pursuit eye movements. J Exp Psychol Hum Percept Perform 32:1136-1154.

Treue S, Maunsell JH (1999) Effects of attention on the processing of motion in macaque middle temporal and medial superior temporal visual cortical areas. J Neurosci 19:7591-7602.

Tsujimoto S, Tayama T (2004) Independent mechanisms for dividing attention between the motion and the color of dynamic random dot patterns. Psychol Res 68:237-244. 